\title{
ОБ ОСНОВНЫХ ПОСТУЛАТАХ СПЕЦИАЛЬНОЙ ТЕОРИИ ОТНОСИТЕЛЬНОСТИ
}

\author{
P. KARD. ERIRELATIIVSUSTEOORIA POHIPOSTULAATIDEST \\ P. KARD. ON THE BASIC PRINCIPLES OF SPECIAL RELATIVITY
}

Хотя обычно второй постулат Эйнштейна понимается в широком. смысле как принцип инвариантности скорости света, правильнее вкладывать в него только смысл отрицания баллистической гипотезы. Иными словами, постулат состоит не более как в утверждении независимости скорости света от движения источника. Поэтому для построения специальной теории относительности достаточно брать в основу принцип относительности, не требуя заранее инвариантности скорости света. В этом смысле в $\left[{ }^{1-3}\right]$ говорилось об обосновании теории относительности без второго постулата Әйнштейна.

Методы подобного обоснования известны уже давно (ссылки см. в $\left.\left[{ }^{1-3}\right]\right)$, но до работ $\left[{ }^{2,3}\right]$ в них использовалось требование, чтобы инерциальные преобразования образовывали группу. Как показано впервые в $\left[{ }^{2,3}\right]$, это требование тоже излишне. В нижеследующем приведем еще один, более простой вариант построения специальной теории относительности на основе принципа относительности и отрицания баллистической гипотезы, но без второго постулата Эйнштейна в широком смысле и без группового постулата.

Рассмотрим два параллельных стержня, движущихся друг относительно друга с параллельной им же скоростью v. Допустим возможную зависимость длины стержня от скорости, выражаемую множителем $g(v)$. Предположим, что левые концы стержней совпадают раньше, а правые позже, и что оба эти события соединены точечным сигналом.

Пусть в системе покоя первого стержня второй движется слева направо, а скорость сигнала равна $u$. Тогда, очевидно,

$$
l_{1} / u=\left[l_{1}-l_{2} g(v)\right] / v,
$$

где $l_{1}$ и $l_{2}$ - длины стержней в покое. В системе покоя второго стержня первый движется справа налево, а скорость сигнала пусть равна $u^{\prime}$. Следовательно,

$$
l_{2} / u^{\prime}=\left[l_{1} g(v)-l_{2}\right] / v .
$$

Исключая из обоих равенств $l_{1} / l_{2}$, находим

$$
(1-v / u)\left(1+v / u^{\prime}\right)=g^{2}(v) .
$$

Эта формула была впервые выведена в [ $\left.{ }^{4}\right]$. Обратим внимание на то, что, хотя в приведенном выводе $v<u$, она справедлива и при $v>u$. В этом убедимся, рассматривая в качестве событий, соединяемых 
точечным сигналом, совпадение правого конца второго стержня с левым концом первого и совпадение левого конца второго стержня с правым концом первого. Тогда вместо (1) получим

$$
l_{1} / u_{1}=\left[l_{1}+l_{2} g(v)\right] / v,
$$

а вместо (2) -

$$
l_{2} / u_{1}^{\prime}=-\left[l_{1} g(v)+l_{2}\right] / v,
$$

причем $v>u_{1}$ и $u_{1}^{\prime}<0$. Исключая опять $l_{1} / l_{2}$, приходим к той же формуле (3) с $u_{1}$ вместо $u$.

Подчеркнем, что формула (3) содержит неявным образом правило преобразования скорости: если в некоторой инерциальной системе скорость равна $u$, то в другой, движущейся в том же направлении со скоростью $v$, она равна $u^{\prime}$.

Поменяем теперь ролями скорости $u$ и $v$. Пусть $v$ будет скоростью сигнала, а $u$ - скоростью другой инерциальной системы. В ней скорость сигнала $v^{\prime}$ будет, очевидно, равна - $u^{\prime}$ (так как скорость другой системы относительно сигнала равна $u^{\prime}$ ). Итак, заменяя в формуле (3) $v \rightarrow u, u \rightarrow v$ и $u^{\prime} \rightarrow-u^{\prime}$, находим

$$
(1-u / v)\left(1-u / u^{\prime}\right)=g^{2}(u) .
$$

Остается исключить $u^{\prime}$ из формул (3) и (6). Результат гласит

$$
v^{-2}\left(1-g^{2}(v)\right)=u^{-2}\left(1-g^{2}(u)\right) .
$$

Но так как $v$ и $u$ независимы, то это равенство возможно только при условии

$$
v^{-2}\left(1-g^{2}(v)\right)=\text { const. }
$$

Если положим постоянную равной нулю, то $g(v)=1$, что дает нерелятивистскую кинематику. В применении к скорости света она приводит или к нарушению принципа относительности (гипотеза эфира), или к зависимости скорости света от движения источника (баллистическая гипотеза). Поэтому постоянную в формуле (8) следует положить равной $c^{-2}$, где $c-$ универсальная скорость, которую в последующем построении теории следует отождествить со скоростью света. Для $g(v)$ получается тогда релятивистская формула

$$
g(v)=\left(1-v^{2} / c^{2}\right)^{1 / 2}
$$

Дальнейшее построение теории общеизвестно.

Итак, основными постулатами специальной теории относительности являются принцип относительности и принцип независимости скорости света от движения источника. В настоящем сообщении предложен простейший, как нам кажется, метод реализации этих принципов.

\section{Л И Т Е Р А У Р А}

1. К а р д П., Изв. АН ЭССР, Физ. Матем., 24, № 4, 449-451 (1975).

2. К а р д П., Изв. АН ЭССР, Физ. Матем., 25, № 3, 227-233 (1976).

3. К а р д П., Изв. АН ЭССР, Физ. Матем., 26, № 4, 389-394 (1977).

4. K a r d, P., Nõukogude Kool, № 3, 225-229 (1975). 